\title{
OnRoute: A Mobile Context-Aware Public Transportation Planning Application
}

\author{
Etienne Bertou and Suleman Shahid \\ Department of Communication and Information Science, Tilburg University, The Netherlands \\ e.m.bertou@tilburguniversity.edu, \\ s.shahideuvt.nl
}

\begin{abstract}
In this paper we describe the design process of OnRoute, a mobile, context-aware travel application for managing navigation with public transport. The application uses an intelligent approach to using location data for easy stepby-step navigation in a more humane way. OnRoute incorporates all means of transportation, including walking, and consistently provides three views with increasing level of detail: a schedule overview, the current position in this step and the next possible action. Furthermore, individual preferences while travelling with public transportation are taken into account. The application learns from users' behavior and proposes better suggestions over time. Userevaluation of the final application design in a real-life setting showed users valued the user experience, design and unique features, and preferred OnRoute to existing smartphone travel applications.
\end{abstract}

Keywords: Public transportation, Route planning, User experience design.

\section{Introduction}

In the Netherlands an up-to-date collection of scheduling data for public transportation has become openly accessible via an open application interface of the travel information organization 9292 [1]. The data makes generating accurate and up-to-date schedules for travelling with multiple means of public transportation possible. When contextual information that becomes relevant during the navigation itself is not incorporated, a pre-defined route provides little benefit to the user. Our aim was to design a better solution for managing navigating with public transportation, focusing on 1) assisting users throughout all phases of travelling, 2) making navigation more user-friendly and 3) providing an overall better travel experience when using public transportation.

\section{User Research}

On different central train stations we interviewed twenty travellers that were waiting for their train to arrive. We discussed currently preferred information sources, problems experienced during navigation with public transportation and desired 
features of a possible solution for making travelling easier. In the Netherlands the railways and stations are all maintained by the Dutch Railways (NS). By interviewing NS-employees working at the station information was gathered on the frequency and nature of questions asked by travellers.

In our user research we identified two main users of public transportation planning applications: commuters and journeyers. Commuters use public transportation to travel along the same route frequently, whereas journeyers navigate along a relatively unfamiliar route to a possibly new destination. We learned commuters mostly value up-to-date information on delays and alternative route planning, while they deem locational information less important. As commuters are already familiar with their route, they prefer a clear overview of their schedule and an estimated time of arrival.

Journeyers, on the other hand, showed more interest in comprehensive route planning information. They would greatly benefit from step-by-step locational and contextual information to go with clear and more detailed instructions. During their trip, they continuously have to gather contextual information for correctly using their predetermined schedule. They are required to search for signs and information boards or ask personnel for information not provided by current route-planning applications. Walking from a train station to the appropriate bus stop or knowing exactly when to get off a bus or train, can be difficult on unfamiliar routes. This was found to result in a less comfortable travelling experience when having to follow a tight, predefined schedule. Users confirmed the importance of including all means of transportation in a travel application. This multi-modality is believed to lead to faster route finding and more comfortable journeys [2]. Accurate and up-to-date information on arrival- and departure times was also considered a primary requirement.

NS staff most frequently assisted travellers in finding the correct platform and confirming transit destinations. Travellers also ask about delays and route alternatives on a regular basis. The interviewed personnel considered answering these questions a major part of their work.

\section{Conceptual Design and Prototyping}

The primary user requirements for our travelling application were deduced from user research. Based on our analysis of the interviews, the process for managing navigation with public transportation could be separated into two major components: planning the initial route and assisting the user during travelling. For route planning many approaches have already been researched. Some schedule planners are able to incorporate much more contextual information [3], while other approaches advocate personalization [4]. For satisfying both commuter's and journeyer's requirements during navigation, we worked on a new solution. We created multiple scenarios and explored possibilities of different approaches to fulfilling user requirements. Examples are using smartphone camera's to overlay locational information or personalizing travel information on screens at train stations and bus stops.

After exploring multiple design alternatives, we decided on developing a smartphone touch-screen application using GPS - much like traditional navigation for 
cars - and a mobile data connection. The application would also feature maps of all Dutch train stations, which are already made available by the NS [5]. We designed an intelligent dashboard for a smartphone application that would use multiple views with an increasing level of information detail to assist both user groups during travelling.

The first dashboard-panel provided an overview of the schedule with the current step in the route being highlighted. It closely resembled the schedule overview of travel apps that are already available, with the exception that it would automatically adapt whenever the route scheduled would no longer be up-to-date or ideal. The second panel contained visual information on progress during the current step with estimated times of arrival and delays. Journeyers would be able to see exactly when and where they were expected to get off the train or buss, which would make travelling much more relaxed. The third and final panel showed specific locational information consisting of point-to-point navigation on how to get to the next point of relevance. Examples are how to get from one train platform to another inside a train station or where to walk after disembarking a bus.

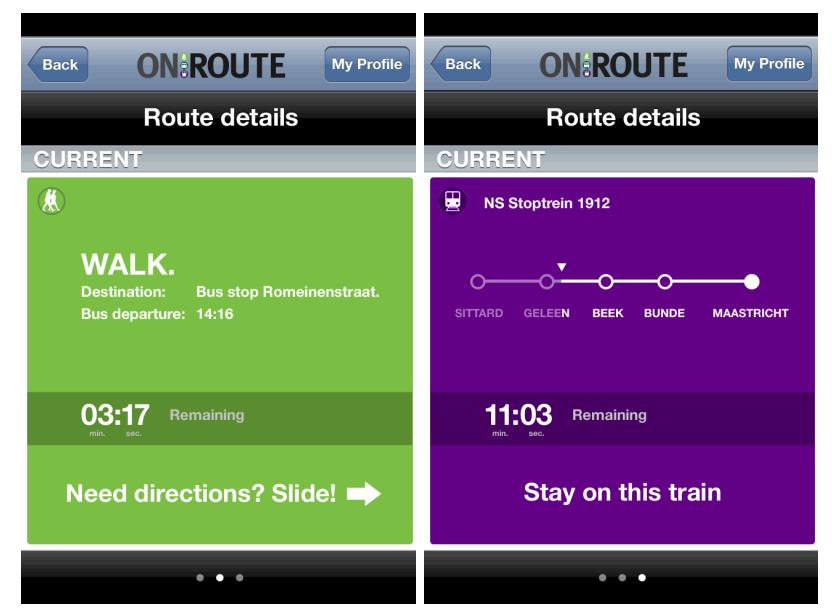

Fig. 1. OnRoute dashboard featuring progress and detailed travel information

OnRoute would personalize advice given during navigation to shift the focus from providing schedule information to creating a better travelling experience. Current route-planning apps rigidly provide the next point and time of departure in the set schedule. Waiting for the time of departure is a major part of travelling with public transportation and could be better spent. The application would provide suggestions based on user preferences and locational data. Instead of waiting at the nearest point of departure, users might prefer walking to the next bus stop in sunny weather and eventually board the same bus. Others might appreciate a cup of coffee or browsing a store. Users could add or remove means of transportation that were incorporated when suggesting routes. Also, they could set some initial customization regarding personal 

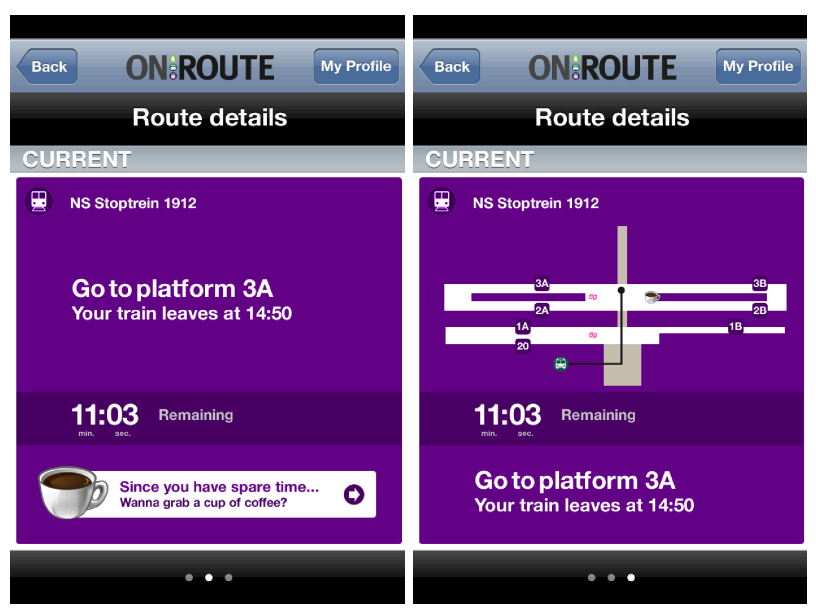

Fig. 2. OnRoute showing personalized suggestions and detailed information for navigation

preferences during navigation. Over time, the application would learn from the behaviour shown by the traveller and adjust suggestions for a pleasurable trip accordingly.

A complete navigational flow for route planning, using the dashboard and setting personalization was determined and all appropriate screens were sketched on paper. To allow task-based evaluation of the early design by the intended end-user, a paper prototype was created. After paper prototype testing, the high fidelity prototype was designed. Users evaluated the application by performing tasks on the actual device. By incorporating the mobile device in the evaluation, we revalidated design choices regarding layout and navigational flow in a realistic context.

\section{$4 \quad$ Evaluation of Final Design}

The final application was designed for iPhone. The fully working application was an extension of the high-fi prototype developed for specific stations and routes and it allowed user evaluation without designers' intervention. This way, we achieved realistic evaluation of the user experience in a real life setting. The application was evaluated by users at two train stations for planning their actual route. The results of early evaluation showed that users valued the overall user experience, minimalistic design and unique approach to assisting with navigating with public transportation. The consistency of separate levels of information on the dashboard panels, the clean interface layouts and the overall visual attractiveness were highly appreciated. All users preferred OnRoute for managing navigation with public transportation to other travel applications they had currently installed on their smartphones. The detailed qualitative and qualitative results of the final usability testing will be discussed in the extended article. 


\section{$5 \quad$ Conclusion and Future Work}

In this paper we discussed the design process of OnRoute, a mobile, context-aware travel application for managing navigation with public transport. By incorporating the intended end-user early and often during the design process, we were able to design an innovative concept for managing navigation with public transportation.

Existing applications focus on route planning, whereas OnRoute also assists in managing navigation with all means of public transportation. It features are a dashboard with multiple levels of detail based on up-to-date locational information and personalizes travelling with public transportation with relevant suggestions. Users valued the comprehensive dashboard, maps of the train stations and natural way of making suggestions during navigation. Providing locational information in several levels of detail and personalizing the trip made navigating with public transportation easier and more pleasurable. Users preferred OnRoute to existing travel applications.

A limitation to our approach is the mobile data usage and use of GPS. Extended use of these features can be expensive and drain the smartphone battery. Alternative techniques for providing the required data are still being explored. Further improvements could be made by researching what specific personal preferences are desirable during navigating. Refining the suggestions provided by OnRoute requires further over-time analysis of actual use during travelling.

Acknowledgements. We would like to thank Eugene Houben, Jan Huntjens, Sylvie Bruys and Romy van Sambeek for working with us in designing the prototypes for OnRoute.

\section{References}

1. REISinformatiegroep B.V.: 9292 gelooft in open data, http://9292opendata.org/ (retrieved)

2. Skoglund, T., Karlsson, M.: Appreciated - but with a fading grace of novelty! Traveller's assessment of, usage of and behavioural change given access to a co-modal travel planner. Social and Behavioral Sciences 48, 932-940 (2012)

3. Vaughn, K.M., Abdel-Aty, M.A., Kitamura, R.: A framework for developing a daily activity and multimodal travel planner. International Transactions in Operational Research 6, 107-121 (1999)

4. Letchner, J., Krumm, J., Horvitz, E.: Trip router with individualized preferences (TRIP): incorporating personalization into route planning. In: Proceedings of the 18th IAAI, vol. 2, pp. 1795-1800 (2006)

5. Nederlandse Spoorwegen: Informatie over station Sittard, http://www.ns.nl/reizigers/reisinformatie/ stationsvoorzieningen/stations/o-s/sittard (retrieved) 